\title{
Construção de uma maquete experimental automatizada para a determinação da constante de Planck com o auxílio da plataforma Arduíno
}

Ivanor Nunes Oliveira ${ }^{1}$

Departamento de Ciências Exatas e Tecnológicas

Universidade Estadual do Sudoeste da Bahia

Vitória da Conquista - BA

Jorge Anderson P. Ramos ${ }^{1}$

Departamento de Ciências Exatas e Tecnológicas

Universidade Estadual do Sudoeste da Bahia

Vitória da Conquista - BA

Wilton Lacerda Silval

Instituto Federal de Educação, Ciência e Tecnologia

Vitória da Conquista - BA

Valteni Douglas Chaves ${ }^{1}$

Instituto Federal de Educação, Ciência e Tecnologia Baiano

Santa Inês- BA

Clênia Andrade de Oliveira Melol

Departamento de Ciências Exatas e Tecnológicas

Universidade Estadual do Sudoeste da Bahia

Vitória da Conquista - BA

\section{Resumo}

Ao modelar um corpo radiante como um conjunto de osciladores harmônicos, o físico Max Planck, em 1900, fez a hipótese de que os osciladores das substâncias podem ter apenas um conjunto discreto de energia e, no processo de interação com o campo de radiação, pode mudar sua energia em porções iguais a hv. A constante de Planck hé

\footnotetext{
${ }^{+}$Construction of an automated experimental device for the determination of the Planck's constant with Arduino platform aid

* Recebido: agosto de 2019.

Aceito: abril de 2020.

${ }^{1}$ E-mails: ivanor_nunes@hotmail.com; jorge@uesb.edu.br; wiltonlacerda@gmail.com; valteni.chaves@ifbaiano.edu.br; clenia@uesb.edu.br
} 
uma das constantes fundamentais da física, juntamente com outras como, por exemplo, a carga e a massa do elétron, a velocidade da luz e a constante de Boltzmann. Como mostra o subsequente desenvolvimento da física quântica, todos os momentos mecânicos dos átomos, moléculas, elétrons e núcleos são expressos em unidades de $h$. Além disso, a constante de Planck entra numa série de relações que desempenham um papel fundamental na física quântica e que determinam os estados discretos das micropartículas, a dualidade onda-partícula de suas propriedades. No presente artigo, propõe-se determinar a constante de Planck através do estudo das curvas características de LASER's de semicondutor, obtidas com o auxílio de uma maquete experimental automatizada que contém uma placa Arduino ligada a um circuito eletrônico com tais dispositivos eletrônicos. A maquete experimental é constituída pelas seguintes partes: banco óptico com suportes; régua com zero central, onde se projetam o máximo principal e máximos secundários do espectro da luz emitida pelo LASER, obtidos com o auxílio de uma rede de difração unidimensional com período conhecido; apontador LASER de semicondutor; placa Arduino Uno com circuito eletrônico que se comunica com o computador via USB; computador. A plataforma Arduino para equipamentos técnicos é recomendada para o processo educacional. Tendo em conta a facilidade de programação e a sua capacidade de estudar processos fisicos em tempo real, propõe-se utilizar placas Arduino na modernização de laboratórios didáticos de Física Geral automatizados manipulados remotamente.

Palavras-chave: Ensino de Física; Laboratório Remoto; Plataforma Arduino.

\begin{abstract}
By modeling a radiant body as a set of harmonic oscillators, the physicist Max Planck in 1900 hypothesized that the oscillators of substances may have only a discrete set of energy, and in the process of interaction with the radiation field, can change its energy in portions equal to hv. Planck's constant $h$ is one of the fundamental constants of physics, along with others such as the charge and mass of the electron, the speed of light, and the Boltzmann constant. As the subsequent development of quantum physics shows, all mechanical moments of atoms, molecules, electrons, and nuclei are expressed in units of $h$. In addition, Planck's constant enters into a series of relations that play a
\end{abstract}


fundamental role in quantum physics and that determine the discrete states of the micro particles, the wave-particle duality of their properties. In this paper, it is proposed to determine Planck's constant by studying the characteristic curves of semiconductor LASERs obtained with the aid of an automated experimental model containing an Arduino board connected to an electronic circuit with these electronic devices. The experimental model consists of the following parts: optical bench with supports; ruler with zero centered, where the main maximum and secondary maximums of the spectrum of the light emitted by the electronic devices obtained with the aid of the diffraction network are projected; one-dimensional diffraction grating with known period; LASER pointer semiconductor; Arduino Uno board with electronic circuit that communicates with the computer via USB; computer. The Arduino platform for technical equipment is recommended for the educational process. Taking into account the ease of programming and its ability to study physical processes in real time, it is proposed to use Arduino boards in the modernization of automated general physics didactic laboratories manipulated remotely.

Keywords: Physics Teaching; Remote Laboratory; Arduino Platform.

\section{Introdução}

Aspectos históricos do surgimento dos conceitos quânticos na física, relacionados com a análise teórica das leis da radiação térmica, podem ser encontrados nos manuais de física das áreas científico-técnicas (BUTIKOV, 1986; EISBERG; RESNICK, 2005; SAVELEV, 1989; SHIPOLSKY, 1974; SIVUKIN, 2008). Como se sabe, inicialmente, esses conceitos foram relacionados com as propriedades da matéria e não com os campos de radiação eletromagnética. Ao modelar um corpo radiante como um conjunto de osciladores harmônicos, o físico Max Planck fez a hipótese de que os osciladores das substâncias podem ter apenas um conjunto discreto de energia $\varepsilon=n h v(n=1,2,3, \ldots)$ e, no processo de interação com o campo de radiação, pode mudar sua energia em porções iguais a $h v$. A constante $h$ é uma constante física fundamental que foi introduzida por ele para descrever a lei da distribuição de energia no espectro de um corpo absolutamente negro e que, mais tarde, recebeu o seu nome. Max Planck, no ano de 1900, obteve a seguinte fórmula para a densidade de energia $\rho(v)$ no intervalo $\mathrm{d} v$ na frequência $v, d u(v)=\rho(v) d(v)$ :

$$
\rho(v)=\left(\frac{8 \pi h v^{3}}{c^{3}}\right)\left\{\frac{1}{e^{\frac{h v}{k T}-1}}\right\},
$$


onde, $h$ é a constante de Planck, $k$ é a constante de Boltzmann. Frequentemente, se usa a constante de Planck reduzida em que $\hbar=\frac{h}{2 \pi}$.

A constante de Planck é uma das constantes fundamentais da física, juntamente com outras como, por exemplo, a carga e a massa do elétron, a velocidade da luz e a constante de Boltzmann. Como mostra o subsequente desenvolvimento da física quântica, todos os momentos mecânicos dos átomos, moléculas, elétrons e núcleos são expressos em unidades de $h$. Além disso, a constante de Planck entra numa série de relações que desempenham um papel fundamental na física quântica e que determinam os estados discretos das micropartículas, a dualidade onda-partícula, ou seja, uma propriedade da natureza, em que, sob uma condição, objetos materiais microscópicos podem exibir propriedades de ondas clássicas e, sob outras condições, propriedades de partículas clássicas (FOCK, 1957). Ao estender as propriedades quânticas dos osciladores das substâncias aos osciladores de campo eletromagnético, pode-se afirmar que a energia do oscilador de radiação toma valores permitidos de $\varepsilon=n h v$. O oscilador de radiação pode, cada vez, adquirir ou perder energia de somente um quantum, ou seja, a quantidade $\Delta \varepsilon=h v$. Se o oscilador for excitado até o enésimo estado quântico, tem a energia $\varepsilon=n h v$ (estritamente, temos que $\varepsilon=n h v+h v$ ) que pode perder em nestágios. Então, do ponto de vista energético, pode-se dizer que existem $n$ quase-partículas, cada uma com energia $h v$. Essas partículas são chamadas fótons partículas eletricamente neutras, portadoras de interação eletromagnética, que possuem um quantum de energia do campo eletromagnético. As principais propriedades do fóton são: 1) É uma partícula do campo eletromagnético; 2) Move-se à velocidade da luz; 3) Existe somente em movimento; 4) É impossível parar um fóton, ele se move a uma velocidade igual à velocidade da luz ou não existe. Das leis da eletrodinâmica clássica segue que o campo de radiação também possui momento linear $\vec{p}$, juntamente com a energia $\mathcal{E}$. Estas grandezas podem ser determinadas pelas equações de Maxwell. Tendo em conta que numa onda luminosa, no vácuo, os vetores $\vec{E}$ e $\vec{H}$ são mutuamente perpendiculares e iguais em módulo, pode-se encontrar a ligação entre energia e momento linear:

$$
\vec{p}=\frac{\varepsilon}{c} \vec{n}
$$

onde $\vec{n}$ é a direção de propagação da onda.

Uma das conclusões fundamentais da teoria da relatividade é a ligação entre a energia e a massa relativística: $\varepsilon=m c^{2}$. Dessa ligação se obtém a massa relativística do fóton, $m$ (a massa de repouso do fóton $m_{o}=0$ ):

$$
m=\frac{h v}{c^{2}} \text {. }
$$

A constante de Planck determina um conjunto amplo de fenômenos físicos do micromundo. Entretanto, pode-se destacar os seguintes métodos de determinação da constante de Planck:

- Através das leis do movimento térmico (CAVALCANTE; HAAG, 2005); 
- Através da balança de Watt, instrumento utilizado para redefinir o quilograma;

- Através dos espectros de raio-X;

- Através dos dados do efeito fotoelétrico externo (OLIVEIRA et al., 2020)

No presente artigo, propõe-se determinar a constante de Planck através do estudo da curva característica de um LASER de semicondutor obtida com o auxílio de uma maquete experimental automatizada que contém uma placa Arduino ligada a um circuito eletrônico. São conhecidos diversos cursos introdutórios ao estudo da plataforma Arduino e suas aplicações em equipamentos técnicos para o processo educacional (NUNES, 2017; RADIONOV, 2011). Devido à facilidade de programação e à sua capacidade de estudar processos físicos em tempo real, queremos utilizar placas Arduino na modernização de laboratórios didáticos automatizados manipulados remotamente (OLIVEIRA et al., 2018). Devido às suas características técnicas e ao fato de que para o estudo da dependência corrente $\times$ tensão do dispositivo eletrônico nomeado acima não há exigência de velocidade, vamos utilizar as placas Arduino Uno e Nano. Placas Arduino mais potentes são aplicáveis para resolver problemas técnicos complexos associados ao desenvolvimento de grandes projetos e sua automação integrada.

\section{Determinação da constante de Planck com o auxílio de uma maquete experimental automatizada}

No presente trabalho, para a determinação da constante de Planck, utiliza-se a maquete experimental descrita na Fig. 1. Com esse objetivo, a maquete contém um circuito eletrônico ligado à plataforma Arduino para a obtenção da curva característica da dependência $I(m A) \times V($ volt $)$ de um LASER de diodo semicondutor. Para a comunicação entre o computador e o Arduino, foi instalado a Macro PLX-DAQ no software Excel, o que permite a obtenção e elaboração dos resultados do experimento em tempo real.

A maquete experimental é constituída pelas seguintes partes: 1) régua com zero central, na qual se projetam o máximo principal e máximos secundários obtidos com o auxílio da rede de difração; 2) rede de difração unidimensional com período conhecido; 3) apontador LASER de semicondutor; 4) placa Arduino Uno com circuito eletrônico que se comunica com o computador via USB; 5) computador.

A rede de difração unidimensional é composta por transparências paralelas para seções de luz da mesma largura, que estão localizadas no mesmo plano. As áreas transparentes são separadas por lacunas opacas. Com a ajuda dessas redes, são feitas observações na luz transmitida. O padrão de difração na rede é o resultado da interferência mútua de ondas provenientes de todas as fendas. 


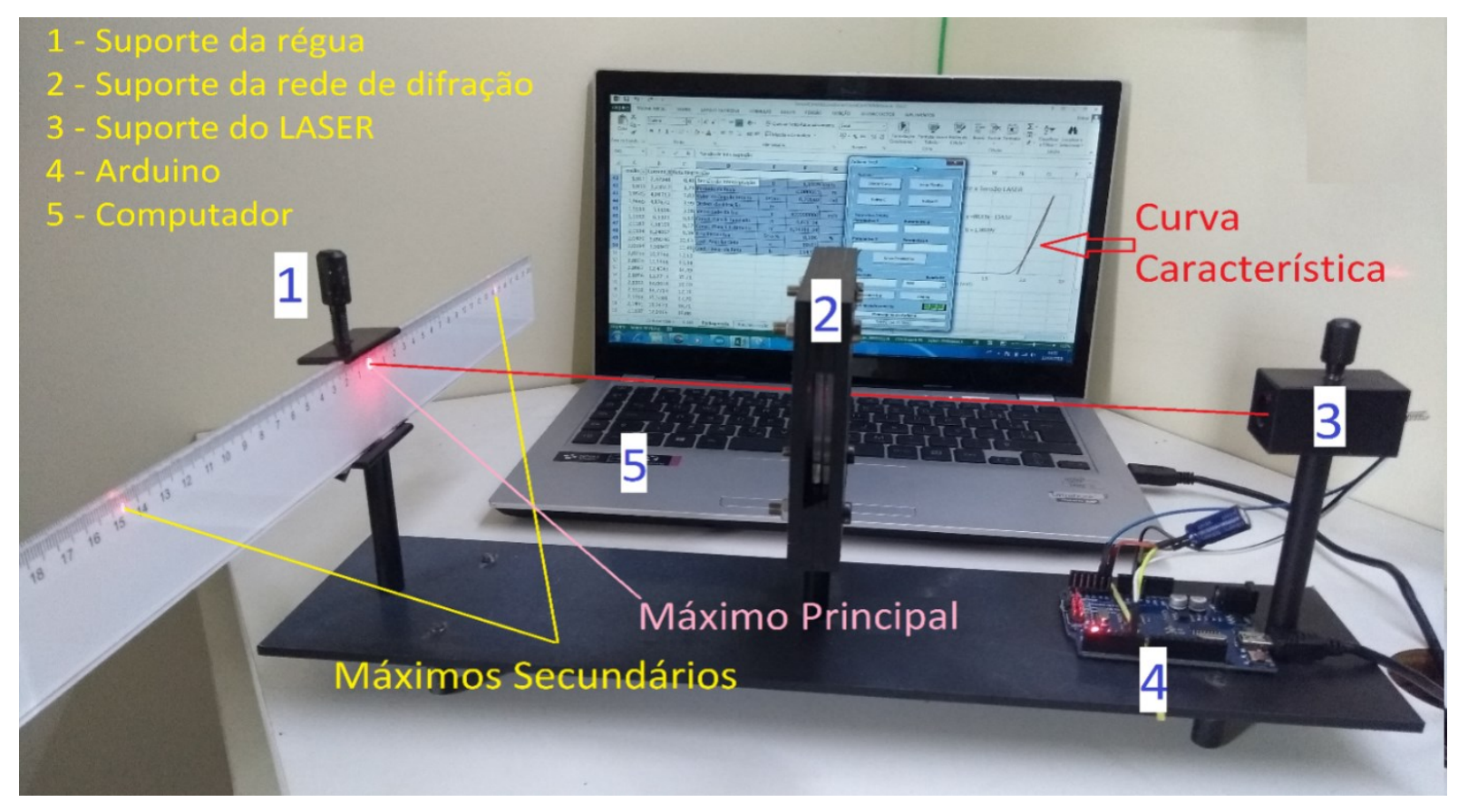

Fig. 1 - Maquete experimental para a determinação da constante de Planck.

\section{II.1 Esquema óptico da maquete experimental}

O esquema óptico da maquete experimental, dado na Fig. 2, mostra a posição do máximo central e de dois máximos secundários do padrão de difração de um LASER de semicondutor (apontador LASER).

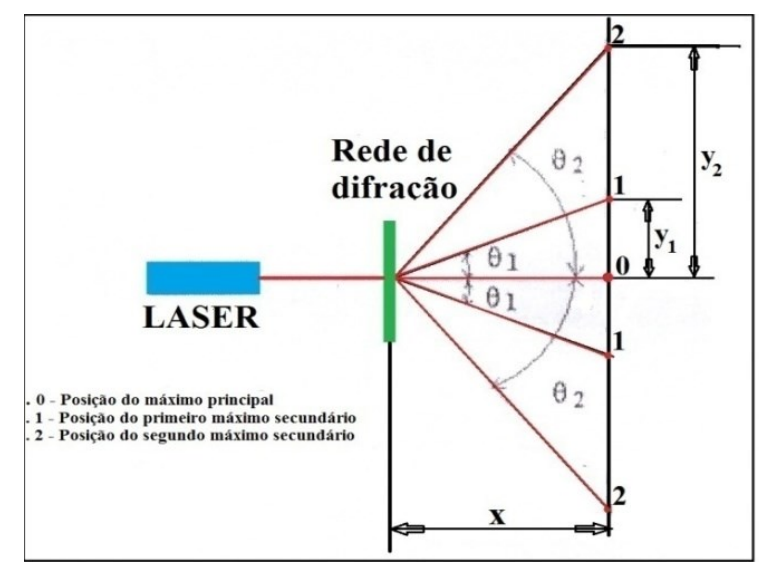

Fig. 2 - Esquema óptico da maquete experimental.

\section{II.2 Obtenção da fórmula de cálculo da constante de Planck}

Utilizando o conceito de energia como medida quantitativa das diferentes formas de movimento da matéria, podemos igualar as expressões quantitativas clássica e quântica da energia: 


$$
e U_{o}=h v,
$$

onde $e$ é a carga do elétron, $U_{o}$ é o potencial que determina o limiar da radiação LASER, $h$ é a constante de Planck e $v$ é a frequência linear da emissão LASER. Logo, obtemos:

$$
h=\frac{e V_{o}}{v}
$$

A expressão da frequência linear tem a seguinte forma:

$$
v=\frac{c}{\lambda}
$$

onde $c$ é a velocidade da luz no vácuo.

Com o auxílio de uma rede de difração com período conhecido, pode-se determinar o comprimento de onda da luz incidente medindo-se um dos ângulos de difração, que contém o máximo principal e um dos máximos secundários das linhas espectrais. A fórmula de cálculo é a condição de formação dos máximos secundários:

$$
d \operatorname{sen} \theta_{m}=m \lambda, \text { ou seja, } \lambda=\frac{d \operatorname{sen} \theta_{m}}{m}, m=1,2,3, \ldots
$$

A partir de (4) e (5), obtemos:

$$
h=\frac{e U_{o} d \operatorname{sen} \theta_{m}}{m c},
$$

onde $d$ é a constante de rede e $m$ é a ordem de difração.

De acordo com o esquema óptico apresentado na Fig. 2, a tangente do ângulo $\theta_{m}$ é calculada pela fórmula $\operatorname{tg} \theta_{m}=\frac{y_{m}}{x}$. O valor do ângulo $\theta_{m}$ é dado pela fórmula: $\theta_{m}=\operatorname{arctg} \frac{y_{m}}{x}$, onde $y_{m}$ é a distância medida, através da régua, desde a ordem zero de difração (máximo principal) até a ordem de difração escolhida (máximo secundário) e $x$ é a distância entre a rede de difração e o ponto de luz criado na régua pelo raio do LASER em ordem zero de difração.

\section{Estudo da dependência corrente/tensão de um LASER de semicondutor utilizando o Arduino}

\section{III.1 Plataforma Arduino - Informações gerais}

São conhecidos diversos microprocessadores e plataformas utilizados para a implementação do gerenciamento de processos físicos aplicados aos sistemas de computação microprocessados. A plataforma Arduino oferece uma série de vantagens em relação a outros dispositivos devido ao seu ambiente de programação simples e claro, preços baixos e uma infinidade de placas de expansão. As placas do Arduino são construídas com base nos microprocessadores Atmel, bem como elementos de comunicação para programação e integração com outros circuitos. 


\section{III.2 O Arduino Uno}

O Arduino Uno é um controlador construído com base no processador ATmega 328. A plataforma possui 14 entradas/saídas digitais (6 das quais podem ser utilizadas como PWM), 6 entradas analógicas, gerador de quartzo de $16 \mathrm{MHz}$, conexão USB, conexão de alimentação, conexão ICSP e botão reset. Para o seu funcionamento é necessário fazer a conexão da plataforma ao computador através do cabo USB ou alimentá-la com a ajuda de um adaptador AC/DC ou bateria. A Fig. 3 mostra a pinagem do Arduino Uno R3 e outros detalhes de hardware da placa (NASIR, 2017).

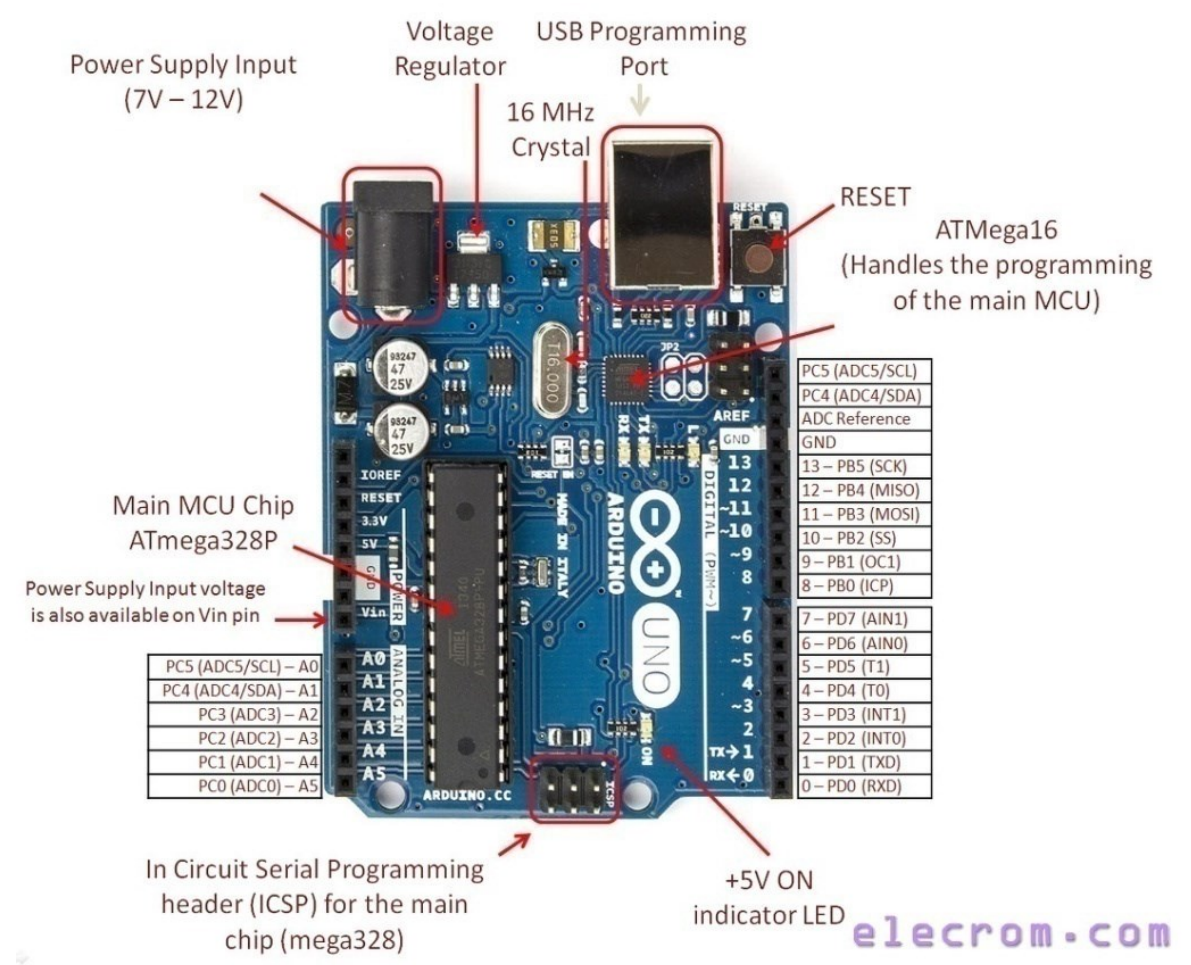

Fig. 3 - Visão geral da placa Arduino Uno (Introduction to Arduino UNO (uses AVR ATmega328)(NASIR, 2017).

\section{Circuito eletrônico para a medição da dependência corrente $\times$ tensão de um dispositivo eletrônico}

Inicialmente, vamos analisar o esquema do circuito de medição dado na Fig. 4. A tensão de alimentação é dada em Ent.1 e Ent.2 e, como resultado, deve ser construído o gráfico da dependência da corrente através do dispositivo eletrônico versus tensão nele:

$$
I_{D} \times U_{D}
$$

ou seja,

$$
\left(U_{\text {saída } 3}-U_{\text {saída 2 }}\right) \frac{1}{R_{1}} \times\left(U_{\text {saída } 1}=U_{\text {saída } 3}\right) .
$$




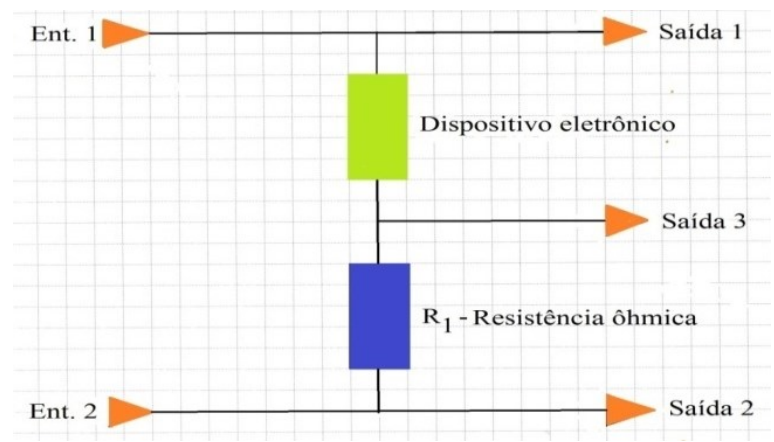

Fig. 4 -Esquema do circuito de medição da dependência corrente $\times$ tensão.

Entretanto, o circuito dado na Fig. 4 não pode ser ligado diretamente ao Arduino. Ele não possui um Circuito Conversor Digital para Analógico (CDA). Para realizar as medições, o circuito de medição é ampliado para que, no circuito resultante, os sinais de entrada possam ser formados por PWM + LPF (Modulação da largura de pulso + RC Filtro passa-baixa), como mostra o esquema dado na seção V, Fig. 7, ou usando um potenciômetro digital/CDA externo. A seguir, são analisados, brevemente, PWM e o Filtro RC passa-baixa e passa alta.

\section{IV.1 PWM}

PWM é a operação de aquisição de um valor analógico variável através de dispositivos digitais. Eles são utilizados para produzir pulsos retangulares - um sinal que muda constantemente entre os valores máximo e mínimo. Este sinal simula uma tensão entre o valor máximo $(5 \mathrm{~V})$ e o mínimo $(0 \mathrm{~V})$, alterando a duração do tempo de ativação de $5 \mathrm{~V}$ em relação à inclusão de $0 \mathrm{~V}$. A duração do valor máximo é chamada de largura de pulso. Para obter diferentes valores analógicos, a largura de pulso é alterada. Com uma mudança suficientemente rápida de períodos ligado-desligado (on-off), um sinal constante entre 0 e $5 \mathrm{~V}$ pode ser aplicado ao laser, controlando assim a intensidade da luz emitida pelo mesmo.

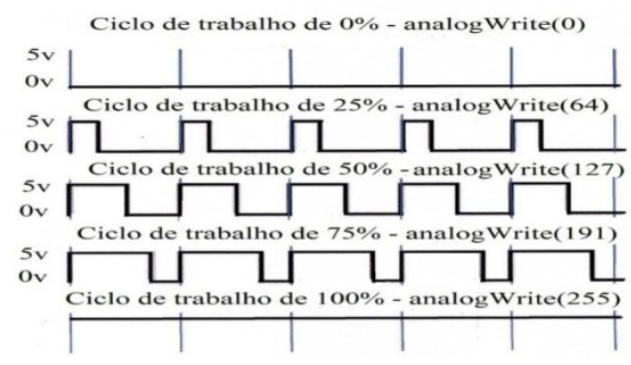

Fig. 5 - Modulação por largura do pulso.

No gráfico da modulação por largura do pulso (Fig. 5), as linhas azuis marcam períodos de tempo $(\mathrm{T})$ permanentes. Como o período é inversamente proporcional à frequência, então, se a frequência de PWM for $500 \mathrm{~Hz}$, as linhas azuis marcarão intervalos 
com duração de 2 milissegundos cada. Chamar a função analogWrite () com uma escala de 0 255 significa que o valor de analogWrite (255) corresponderá a um ciclo de trabalho de 100\% (5V permanente ligado), logo, por exemplo, o valor de analogWrite (127) será 50\% do ciclo de trabalho.

Normalmente, a amplificação da emissão laser é regulada com modulação por largura de pulso (PWM). No entanto, para a obtenção da curva $\mathrm{I} \times \mathrm{V}$, realmente, precisamos aplicar uma tensão analógica constante e corrente no dispositivo eletrônico. Isso pode ser feito filtrando-se a saída PWM com um filtro resistor-capacitor de baixa passagem.

\section{IV.2 Filtros RC - Filtro passa-baixa e filtro passa-alta}

Os filtros são circuitos eletrônicos que deixam passar uma determinada banda de frequência sem atenuação e suprimem todas as outras frequências. A frequência na qual a supressão começa é chamada de frequência de corte $f_{c}$.

O mais simples entre os filtros é o filtro RC. O princípio de sua operação baseia-se no fato de que à medida que a frequência muda, a reatância do capacitor muda inversamente proporcional à frequência, enquanto a resistência do resistor permanece inalterada. No diagrama da Figura 6a, um capacitor é conectado em série com um resistor. Quando um sinal de baixa frequência é alimentado à entrada de tal filtro, a reatância do capacitor $\mathrm{C}$ será muito maior que a resistência do resistor R. Como resultado, a queda de tensão $V_{C}$ no capacitor será grande e no resistor $V_{R}$ pequena. Ao aplicar um sinal de alta frequência à entrada deste filtro, a imagem será invertida: $V_{C}$ será pequeno e $V_{R}$ será grande.

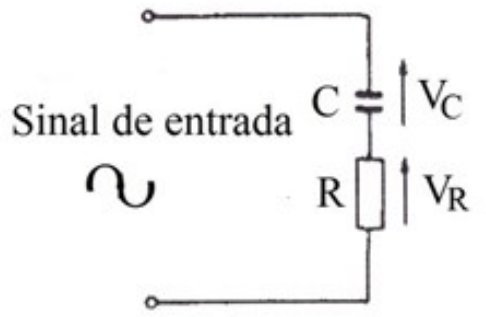

Fig. 6a

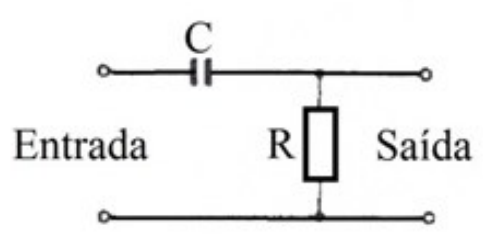

Fig. $6 \mathrm{~b}$

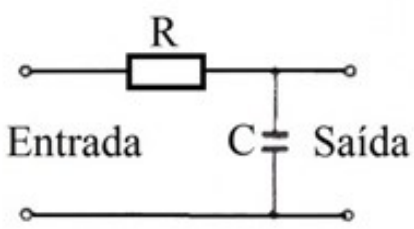

Fig. 6c

Fig. $6 a, 6 b, 6 c-$ Filtros $R C$.

Se, agora, apresentarmos este esquema, como na Fig. 6c, onde a queda de tensão através do capacitor é uma saída, então os componentes de baixa frequência prevalecerão no sinal de saída, e os componentes de alta frequência serão bastante atenuados. Em outras palavras, temos um filtro passa-baixa. Por outro lado, se a tensão de saída é obtida no resistor (Fig. 6b), então obtemos um filtro passa-alta. Os valores de $\mathrm{R}$ e $\mathrm{C}$ determinam a frequência de corte do filtro. 


\section{Esquema de circuito eletrônico com a utilização da saída analógica PWM + LPF para o estudo do dispositivo eletrônico LASER de semicondutor com o Arduino}

Para a obtenção da curva característica do dispositivo eletrônico em estudo, foi tomado esquema de circuito eletrônico mostrado na Fig. 7.

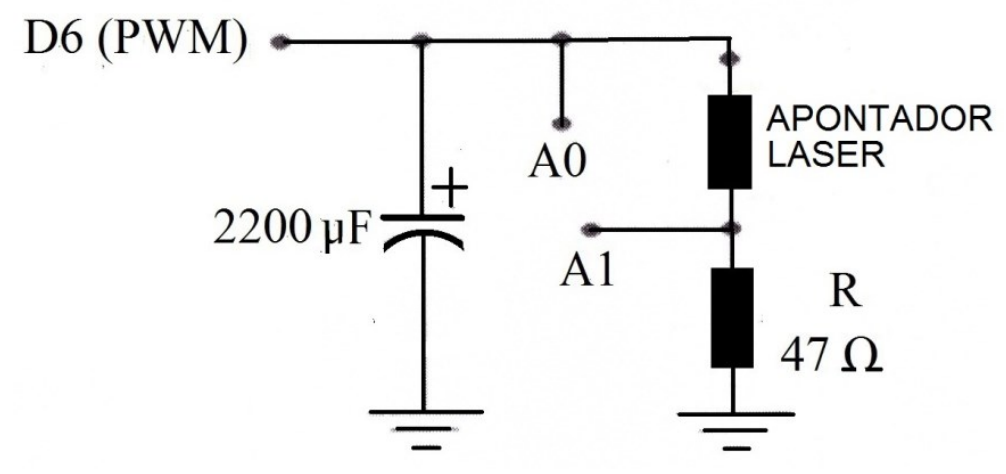

Fig. 7 - Esquema para medição de $I \times V(N U N E S, 2017)$.

Os componentes eletrônicos utilizados foram:

- 1 capacitor eletrolítico de $2200 \mu F$;

- 1 resistor de 47 Ohm;

- 1 apontador LASER.

\section{A automatização do experimento}

\section{VI.1 O hardware}

A medição da curva característica $\mathrm{I} \times \mathrm{V}$ de um LASER de semicondutor para a determinação da constante de Planck foi realizada de acordo com o esquema eletrônico dado na Fig. 7. Esse componente eletrônico tem sido utilizado no estudo dos fenômenos da luz coerente e em experimentos de óptica ondulatória dos Laboratórios Didáticos de Óptica. Como se sabe, a coerência é um dos conceitos mais importantes de óptica e significa a capacidade da luz para exibir fenômenos de interferência. A luz é chamada coerente quando há uma relação de fase fixa entre a intensidade do campo eletromagnético em diferentes pontos no espaço ou em momentos diferentes. O espectro da difração do feixe luminoso emitido pelo LASER ao incidir na rede de difração pode ser observado, diretamente, num anteparo (régua) ou com o auxílio de uma Web-câmera instalada na maquete (Fig. 1) sem a necessidade de escurecer o ambiente.

A montagem do circuito eletrônico é dada na Fig. 8. O LASER encontra-se ligado em série com o resistor limitador de corrente. No caso deste experimento, o LASER utilizado foi retirado de um apontador laser que já possui um resistor de $63 \mathrm{ohms} \mathrm{instalado.}$ 
O terminal negativo (-) do capacitor e um dos terminais do resistor encontram-se conectados ao terminal de referência da placa Arduino (GND). Já os terminais positivos (+) do LASER e do capacitor são conectados ao pino D6 da placa Arduino. Deve-se salientar que este pino, D6, foi configurado por software para operar como uma saída PWM (Pulse Width Modulation). Como o sinal PWM fornece uma onda de corrente/tensão pulsada, foi necessário convertê-la numa saída analógica linear. Para tanto, foi utilizado um capacitor, cuja função principal é atuar como filtro. Desta forma, é possível produzir um sinal de corrente/tensão analógico com regulação efetuada via software. Finalmente, o terminal negativo do LASER é conectado ao outro terminal do resistor limitador de corrente.

Para encontrar a corrente e tensão presentes no LASER foi adotada a seguinte metodologia:

- A entrada analógica $A_{1}$ do Arduino é utilizada para medir a tensão sobre o resistor, enquanto a entrada analógica $A_{0}$ utilizada para medição da tensão total do circuito, ou seja, a tensão fornecida pela saída PWM no pino D6. Assim, é possível determinar a tensão sobre o LASER subtraindo da tensão obtida na entrada $A_{0}$ aquela encontrada na entrada $A_{1}$. Logo: $V_{\text {laser }}=\left(\mathrm{A}_{0}-\mathrm{A}_{1}\right) V$.

- Para obtenção do valor da corrente no LASER, foi utilizada a tensão obtida na entrada $A_{1}$, pois com ela é possível calcular a corrente que percorre o resistor utilizando a lei de Ohm. Como este resistor encontra-se ligado em série com o LASER, a sua corrente é a mesma do resistor. A expressão utilizada para o cálculo é: $I=\frac{V_{\mathrm{A} 1}}{63 \Omega}$.

- Finalmente, utilizando uma interface que permite a comunicação do Arduino com o software Excel o gráfico da curva corrente $\times$ tensão do LASER pode ser obtida no Excel.

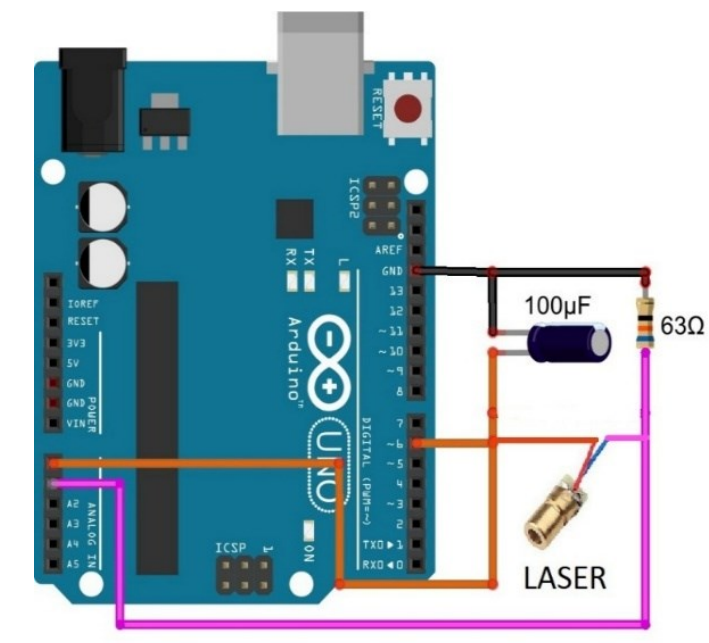

Fig. 8 - Montagem do circuito eletrônico ligado ao Arduino Uno.

\section{A comunicação do Arduino com o computador}

Queremos transferir dados das medições do Arduino para o Excel, um editor de planilhas da Microsoft que possui ferramentas de cálculo e construção de gráficos. 
VII.1 Procedimentos necessários para transferir dados das medições do Arduino para o Excel

1 - Escrever o programa para o Arduino (sketch) para transferir os dados das medições para o Excel;

2 - Instalar um programa, uma Macro PLX-DAQ, no computador, que vai permitir que o Excel receba os dados do Arduino.

\section{VII.2 O programa para o Arduino}

Abaixo é dado o programa para o Arduino em conformidade com o esquema eletrônico escolhido.

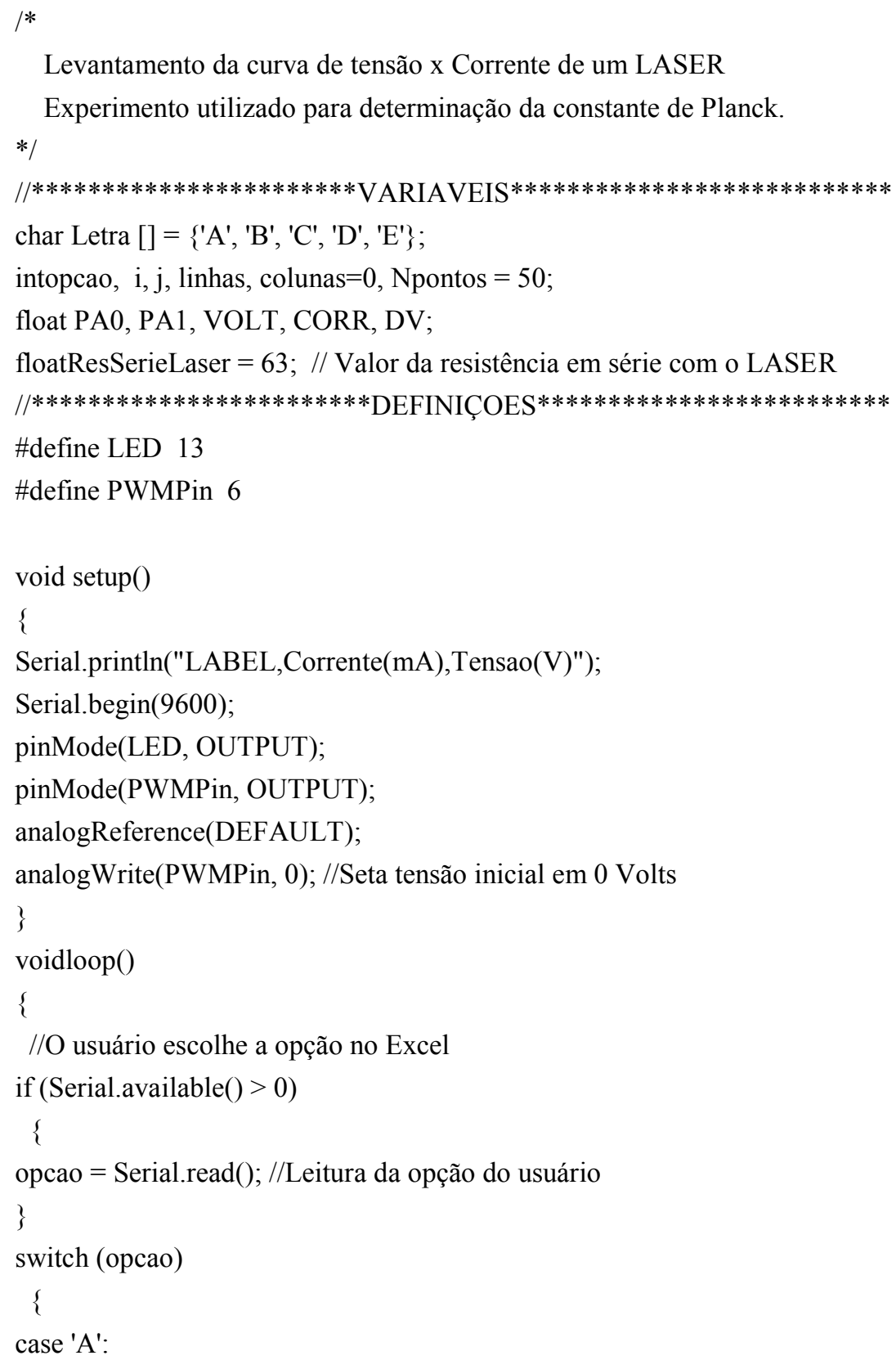


linhas $=1$;

Serial.println("RESETROW");

digitalWrite(LED, HIGH); //Liga o Led indicativo do Arduino

for (int $\mathrm{i}=5 ; \mathrm{i}<=245 ; \mathrm{i}=\mathrm{i}+3$ )

\{

analogWrite(PWMPin, i); //Definição da taxa do PWM

delay(100); //Tempo de estabilização do sinal

// Faz uma média de Npontos do sinal capturado

$\mathrm{PA} 0=0$

for $(j=0 ; j<$ Npontos $; j++)$

\{

$\operatorname{delay}(2)$;

$\mathrm{PA} 0=\mathrm{PA} 0+$ analogRead $(0) ; / /$ Leitura da porta A0 do arduino

\}

PA0 = PA0 / Npontos; // Valor médio de Npontos medidos (FILTRO)

// Faz uma média de Npontos do sinal capturado (Filtro)

$\mathrm{PA} 1=0$

for $(\mathrm{j}=0 ; \mathrm{j}<$ Npontos; $\mathrm{j}++)$

\{

$\operatorname{delay}(2)$;

PA1 $=$ PA1 + analogRead(1);//Leitura da porta A1 do arduino

\}

PA1 = PA1 / Npontos;// Valor médio de Npontos medidos (FILTRO)

$\mathrm{DV}=(\mathrm{PA} 0$ - PA1 $) ; / /$ Tensão sobre o LASER em bits

$\mathrm{CORR}=(5000 * \mathrm{PA} 1 / 1023) /$ ResSerieLaser; //Corrente LASER em Ampere

VOLT $=(5.0 *$ DV / 1023); //Tensão no LASER em Volts

linhas $=$ linhas +1 ; //ncrementa o contador de colunas do Excel

// Transfere a informação de tensão do Arduino para o Excel

Serial.print("CELL,SET,");

Serial.print (Letra [colunas]);

Serial.print (linhas);

Serial.print(",");

Serial.println (VOLT, 4); //4 casas decimais

// Transfere a informação de corrente do Arduino para o Excel

Serial.print("CELL,SET,");

Serial.print (Letra [colunas +1$]$ );

Serial.print (linhas);

Serial.print(",");

Serial.println (CORR, 5); //5 casas decimais

delay $(50)$;

\}

digitalWrite(LED, LOW); //Apaga o LED indicativo do Arduino

opcao $=100 ; / /$ Cria um valor de opção inválido

break;

case 'B': 


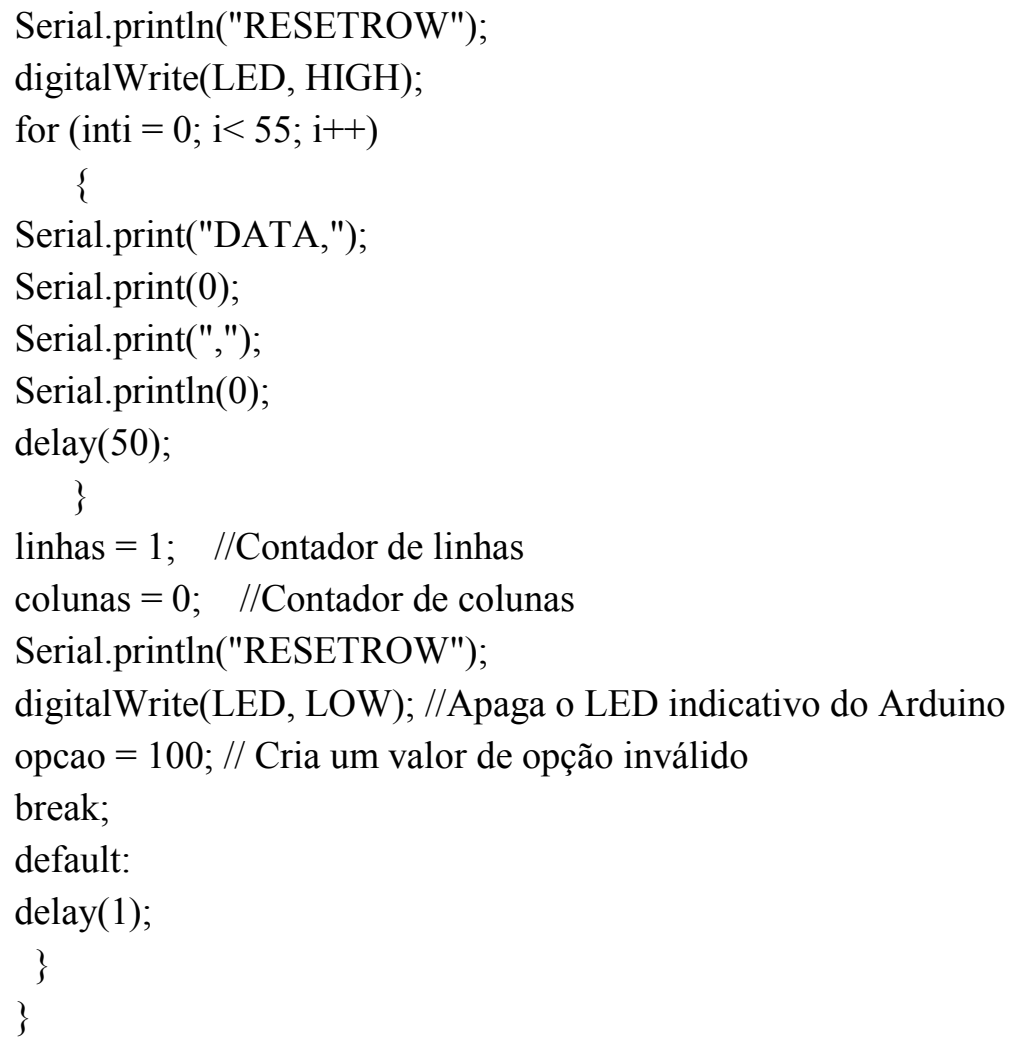

\section{Apresentação dos resultados das medições}

\section{VIII.1 A regressão linear}

Para a obtenção da tensão limiar $U_{0}$ da emissão de radiação LASER, torna-se necessário aplicar o método dos mínimos quadrados para ajustar a contribuição da parte quase retilínea das medições no gráfico através da regressão linear. O ajuste da curva obtida é dado pela função $y=a x+b$, equação da reta (variação linear). Omitindo-se os cálculos matemáticos, as expressões para o parâmetro a e para o parâmetro b procurados têm as seguintes formas:

$$
a=\frac{n \sum_{i=1}^{n} x_{i} y_{i}-\sum_{i=1}^{n} x_{i} \sum_{i=1}^{n} y_{i}}{n \sum_{i=1}^{n} x_{i}^{2}-\left(\sum_{i=1}^{n} x_{i}\right)^{2}} ; \quad b=\frac{1}{n}\left(\sum_{i=1}^{n} y_{i}-a \sum_{i=1}^{n} x_{i}\right) .
$$

$\mathrm{O}$ valor do erro médio quadrático da aproximação examinada também pode ser obtido com o auxílio da teoria dos erros de medição das grandezas físicas (OLIVEIRA, 2018). Entretanto, utilizamos as ferramentas de cálculo e construção de gráficos do Excel na elaboração dos resultados. Foram realizadas medições da dependência I $(\mathrm{mA}) \times \mathrm{V}(\mathrm{v})$ do dispositivo eletrônico: Apontador LASER de semicondutor e, com o auxílio do Excel, foi obtida a curva característica do referido dispositivo, mostrada abaixo. 


\section{VIII.2 Gráficos da curva característica obtida com o Excel}

A solução utilizada para comunicação da plataforma Arduino com o software Excel foi a ferramenta Parallax Data Acquisition tool (PLX-DAQ). Esta ferramenta de software é uma add-in gratuita que pode ser agregada ao Microsoft Excel. Com ela, é possível estabelecer uma comunicação serial bidirecional do Computador com um Microcontrolador.

O PLX-DAQ recebe e organiza os dados enviados pelo microcontrolador em linhas e colunas dentro de uma planilha Excel, tudo isto em tempo real. Depois de instalado, o PLXDAQ pode receber várias customizações. Na Fig. 9 é apresentada a customização efetuada e utilizada neste trabalho.

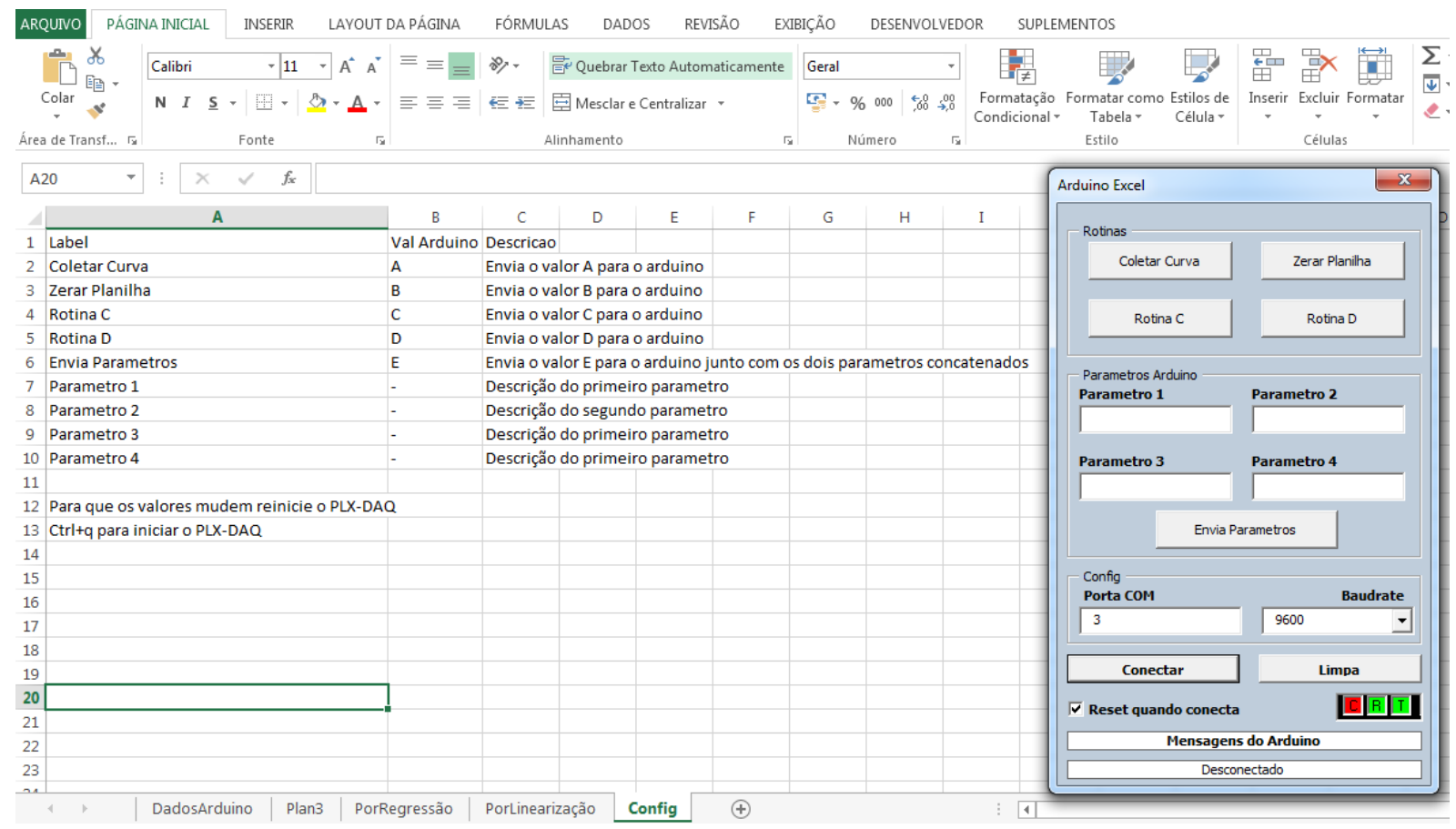

Fig. 9 - Configuração utilizada no PLX-DAQ.

Para ativar o PLX-DAQ basta pressionar as teclas Ctrl + Q na planilha Excel. Como pode ser notado no código do programa instalado no Arduino, somente dois botões foram necessários para este experimento. O botão denominado "Coletar Curva", o qual é responsável por enviar o caractere " $A$ " para o Arduino. No Arduino, uma vez que este caractere é recebido, ele é armazenado na variável intitulada opção. Desta forma, o fluxo de código toma a decisão de coletar os dados de corrente e tensão do laser, enviando-os para a planilha Excel. Esta rotina é parcialmente apresentada na Fig. 10. Caso o botão denominado "Zerar Planilha" seja selecionado no PLX-DAQ, o caractere "B" será enviado para o Arduino. E, assim, todos os dados da planilha serão apagados. Um trecho da rotina de código do programa instalado no microcontrolador da plataforma Arduino que executa esta operação é apresentado na Fig. 11. 


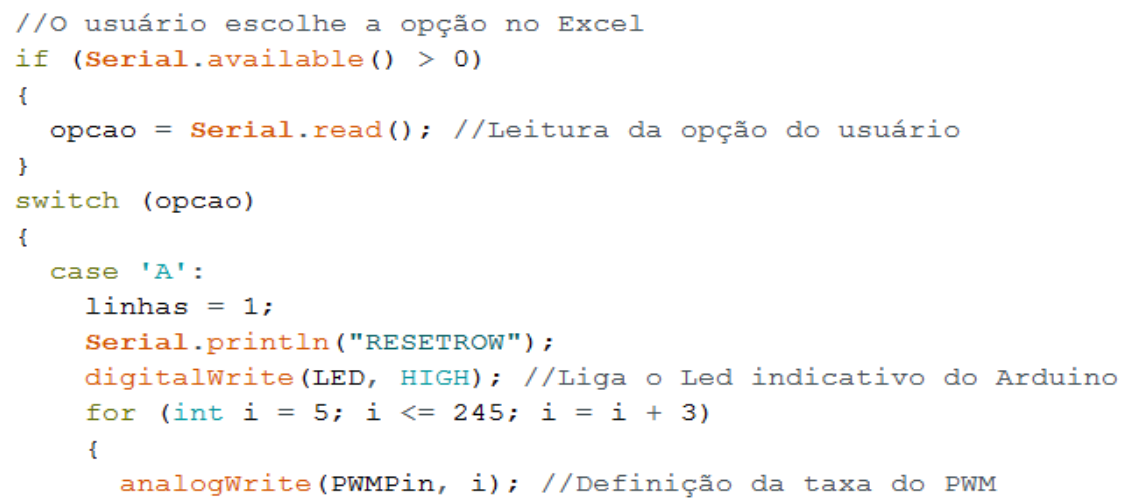

Fig. 10 - Código para escolha da captura de dados e envio para a planilha Excel.

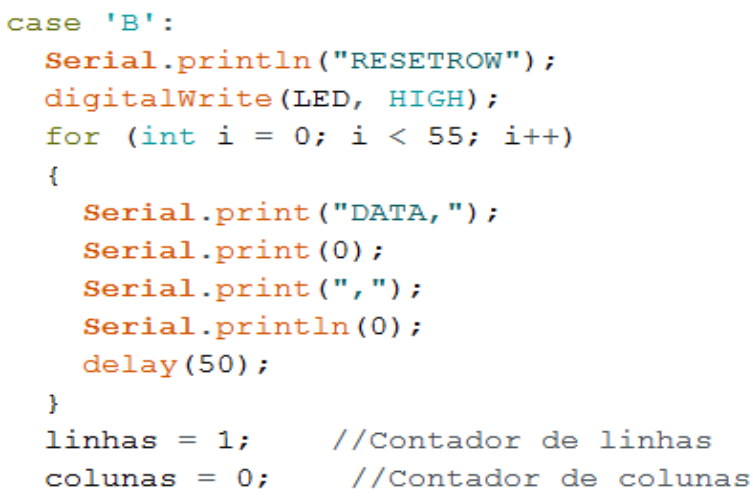

Fig. 11 - Código para escolha da remoção dos dados da planilha Excel.

De forma simplificada, o processo de obtenção da curva Corrente versus Tensão no LASER se dá da seguinte maneira: uma vez que a opção "Coletar Curva" for escolhida, o Arduino iniciará o processo de aumentar passo a passo o comprimento do pulso PWM, e consequentemente a tensão de alimentação do circuito no qual o LASER encontra-se interligado.

Os valores analógicos de tensão sobre o LASER e resistor são obtidos. Para minimizar ruídos característicos da conversão analógico digital, foi implementado um filtro digital passa baixa utilizando a operação de média. Os dados de corrente e tensão do LASER, depois de tratados, são enviados para a planilha Excel.

O gráfico da curva característica Corrente $\times$ Tensão é então plotado em tempo real. Os dados resultantes desta operação estão apresentados na Fig. 12. Na Fig. 13 encontram-se a curva característica do LASER e a regressão linear. 


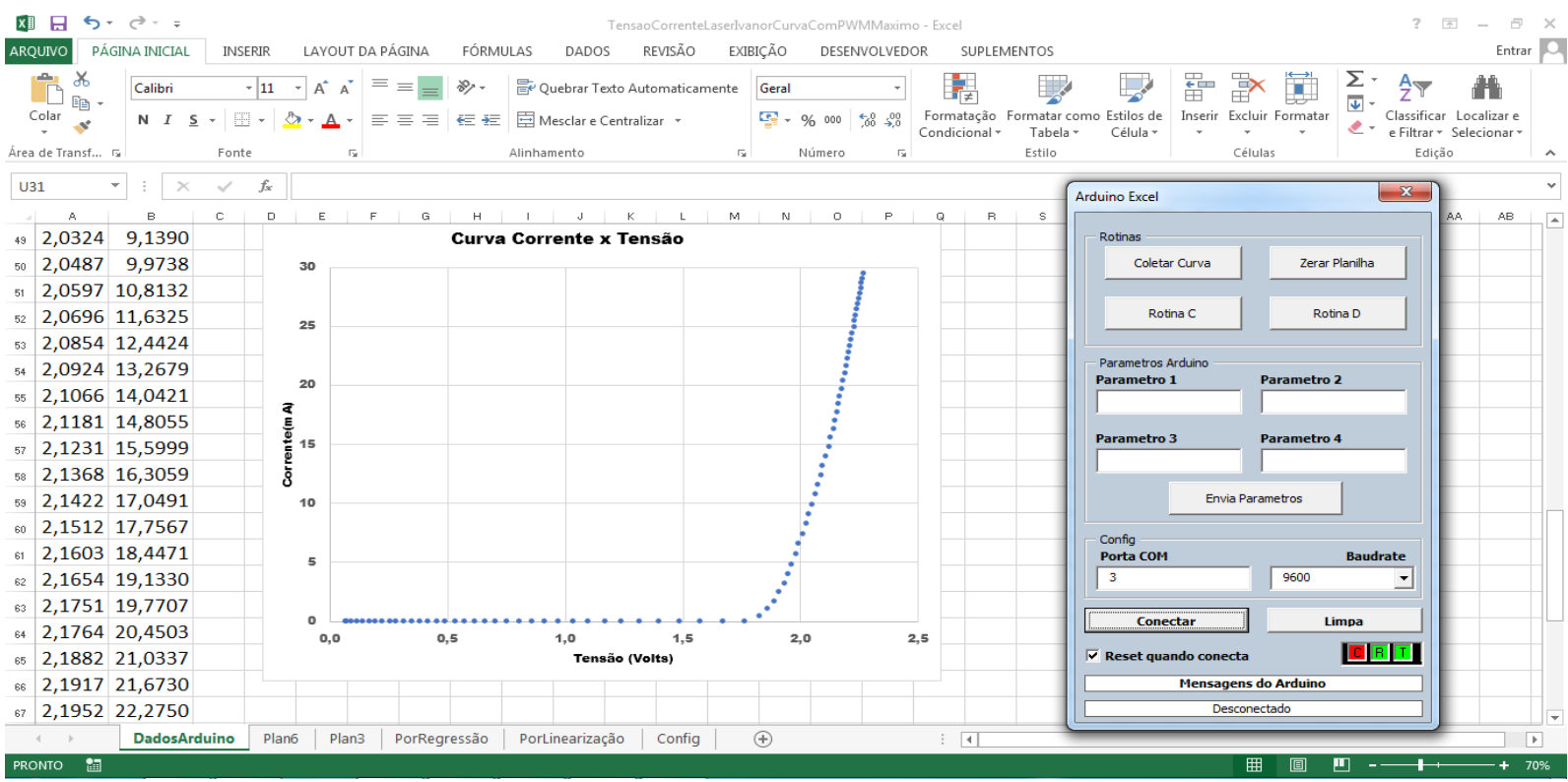

Fig. 12 - Curva característica do LASER $-I \times V$.

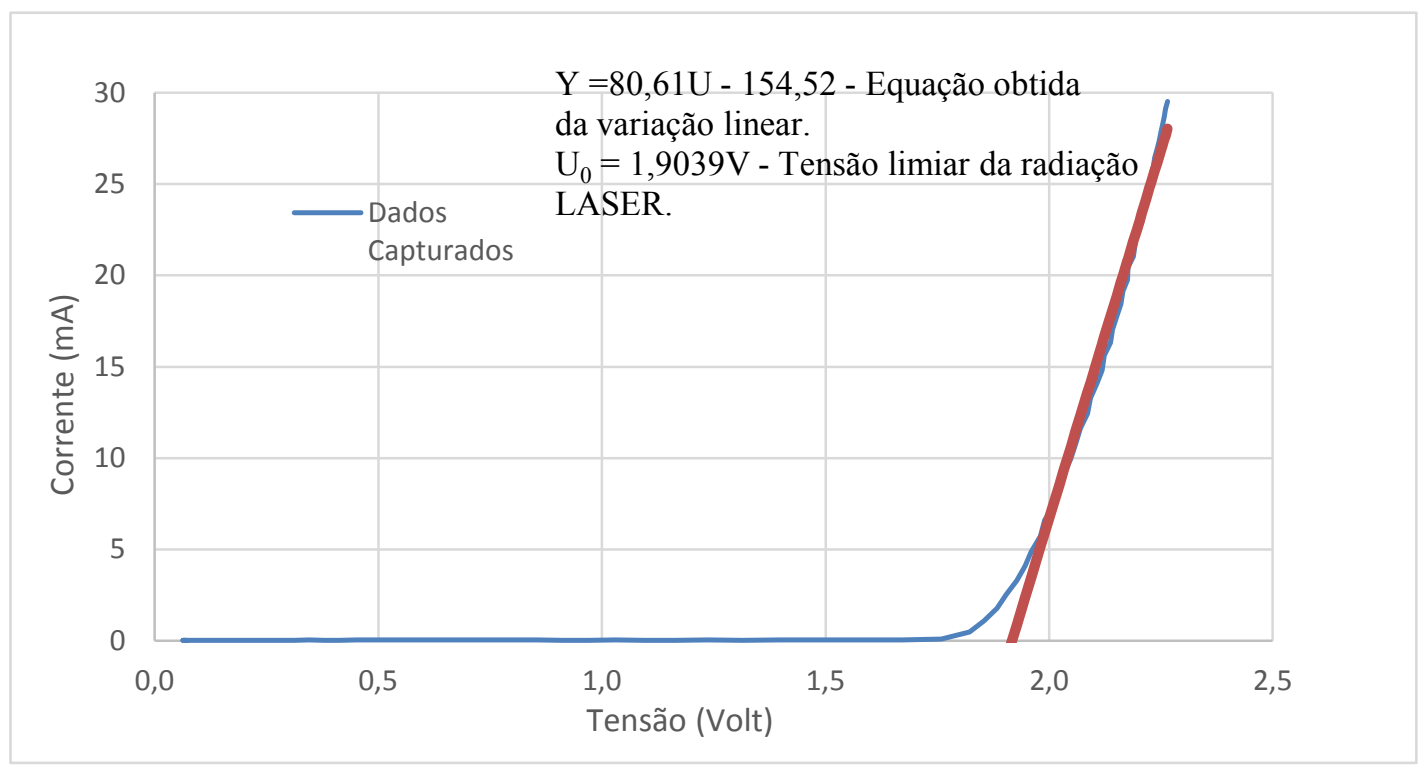

Fig. 13-Curva característica do LASER $-I(m A) \times V($ volt $)$ e regressão linear.

\section{VIII.3 A determinação do valor da constante de Planck}

O cálculo da constante de Planck é realizado com o auxílio da expressão (8) obtida na seção II.2:

$$
h=\frac{e U_{o} d \operatorname{sen} \theta_{m}}{m c} .
$$

Com o auxílio das ferramentas de cálculo do software Excel foram obtidos os seguintes resultados: 
Tabela 1 - Resultados obtidos com o auxílio das ferramentas de cálculo do Excel.

\begin{tabular}{|c|c|c|c|}
\hline Denominação & Representação & Valor & Unidade \\
\hline Tensão limiar de radiação & $U_{o}$ & 1,904 & Volt \\
\hline Período da rede de difração & $d$ & $1 \times 10^{-6}$ & $m$ \\
\hline Valor do ângulo de difração & $\vartheta_{m} ; m=1$ & 0,706 & rad \\
\hline Velocidade da luz & $c$ & $3,00 \times 10^{8}$ & $\mathrm{~m} / \mathrm{s}$ \\
\hline $\begin{array}{l}\text { Constante de Planck (valor } \\
\text { tabelado) }\end{array}$ & $h$ & $6,626 \times 10^{-34}$ & $J . S$ \\
\hline $\begin{array}{l}\text { Constante de Planck (valor } \\
\text { estimado) }\end{array}$ & $h^{\prime}$ & $\begin{array}{r}(6,594 \pm 0,004240) \\
\times 10^{-34} \\
\end{array}$ & $J . s$ \\
\hline Erro relativo percentual & Erro relativo \% & 0,483 & $\%$ \\
\hline Carga do elétron & $e$ & $-1,602 \times 10^{-19}$ & $C$ \\
\hline Coeficiente angular da reta & A & 80,61 & \\
\hline Coeficiente linear da reta & $\mathrm{B}$ & $-154,52$ & \\
\hline
\end{tabular}

O cálculo do ângulo de difração $\theta_{1}=0,706$ rad foi realizado de acordo com os valores medidos, durante o experimento, de $y_{1}=14,4 \mathrm{~cm}$, distância desde o máximo principal até o primeiro máxímo secundário, e de $x=16,9 \mathrm{~cm}$, distância desde a rede de difração até o máximo principal, em conformidade com a expressão $\theta_{1}=\operatorname{arctg} \frac{y_{1}}{x}$.

Observando-se os resultados contidos na Tabela 1 , pode-se ver que o valor estimado encontrado para a constante de Planck $h^{\prime}=(6,594 \pm 0,004240) \times 10^{-34} \mathrm{~J} . \mathrm{s}$, o que implica em um erro relativo percentual de $0,0643 \%$.

\section{Considerações finais}

Para determinação da constante de Planck foi construída uma maquete experimental automatizada (Fig. 1) constituída pelos seguintes componentes: 1) régua com zero central, na qual se projetam o máximo principal e máximos secundários obtidos com o auxílio da rede de difração; 2) rede de difração unidimensional com período conhecido; 3) apontador LASER de semicondutor; placa Arduino Uno com circuito eletrônico que se comunica com o computador via USB; 4) computador. Como se sabe, diversos microprocessadores e plataformas têm sido utilizados para a implementação do gerenciamento de processos físicos aplicados aos sistemas de computação microprocessados. Entretanto, verifica-se o crescimento do uso da plataforma Arduino no processo educacional por oferecer uma série de vantagens em relação a outros dispositivos devido ao seu ambiente de programação simples e claro, preços baixos e uma infinidade de placas de expansão. Nesse sentido, a nossa atenção tem sido voltada para os novos modelos educacionais de interação entre teoria e prática 
surgidos em função da difusão e do avanço das Novas Tecnologias da Informação e Comunicação - NTIC's. Assim, a maquete experimental automatizada para a determinação da constante de Planck teve os sistemas eletrônico e de software que controlam a maquete atualizados.

A principal mudança no sistema de controle é a interface de comunicação com o computador, onde essa nova comunicação é baseada no protocolo USB. Esse novo sistema é baseado no microcontrolador ATMEL328 (Atmel, 2015) por meio da plataforma Arduino ${ }^{2}$. É importante observar que essa plataforma é um projeto de circuito de código aberto, baseado em microcontrolador da indústria Atmel. Uma placa física em código aberto baseada em um circuito de entradas e saídas simples. A mesma já possui conversor analógico/digital. Assim, é possível gerenciar o controle da maquete automatizada usando a interface USB do computador, por meio de um dispositivo de baixo custo. A escolha do LASER de semicondutor no experimento para a determinação da constante de Planck é interessante pela sua importância como aparelho da optoeletrônica. Esse componente eletrônico tem sido utilizado no estudo dos fenômenos da luz coerente em experimentos de óptica ondulatória dos Laboratórios Didáticos de Óptica. Como se sabe, a coerência é um dos conceitos mais importantes de óptica ondulatória e significa a capacidade da luz para exibir fenômenos de interferência. Neste sentido, a plataforma Arduino, pode ser utilizada tanto na automatização de outros experimentos de física moderna (por exemplo, o estudo do espectro de radiação do LASER, o estudo do diagrama direcional da sua radiação, etc.), como também na automatização de experimentos didáticos tradicionais, relacionados com os fenômenos de interferência e difração, onde o LASER de semicondutor encontra aplicação.

A experiência de ensino na Universidade Estadual do Sudoeste da Bahia (UESB) mostra que a automatização dos laboratórios de Física Geral em desenvolvimento nessa IES pode resolver um problema central do Curso de Licenciatura em Física, modalidade a distância, a realização de aulas práticas laboratoriais de Física Geral com experimentos que podem ser manipulados à distância via Internet.

\section{Referências}

BUTIKOV, E. I. Óptica. Moscou, Rússia: Editora: “Escola Superior,” 1986.

CAVAlCANTE, M. A.; HAAG, R. Corpo negro e determinação experimental da constante de Planck. Revista Brasileira de Ensino de Física, v. 27, n. 3, p. 343-348, 2005.

EISBERG, R.; RESNICK, R. Radiação térmica e o postulado de Planck. In: Física Quântica. Rio de Janeiro: Editora Campus, 2005. p. 17.

\footnotetext{
${ }^{2}$ Site oficial do Arduino: <https://www.arduino.cc/>.
} 
FOCK, V. A. Sobre a Interpretação da Mecânica Quântica, v. 62, n. 8, p. 466, 1957.

NASIR, S. Z. Introduction to Arduino UNO (uses AVR ATmega328). Disponível em: $<$ https://www.theengineeringprojects.com/2017/08/introduction-to-atmega328.html>.

NUNES, L. A. de O. 29_Curva de Tensão Corrente de LEDs. oficiencia. Disponível em: $<$ https://www.youtube.com/watch?v=nJaQxY6yl9Y>.

OLIVEIRA, I. N. DE et al. Estudo das propriedades do Diodo Emissor de Luz ( LED ) para a determinação da constante de Planck numa maquete automatizada com o auxílio da plataforma Arduíno. Revista Brasileira de Ensino de Física, v. 42, n. e20190105, 2020.

OLIVEIRA, I. N. et al. Automatização de laboratórios didáticos de física geral - Construção de uma maquete experimental automatizada para a determinação da constante de Planck. Experiências em Ensino de Ciências, v. 13, n. 1, p. 131-153, 2018.

OLIVEIRA, I. N. Erros de medição das grandezas físicas e elaboração dos resultados do experimento. [s.1.] Edições UESB, 2018.

RADIONOV, A. A. Equipamentos elétricos e eletroautomaticos. Magnitogorsk: Editora da Universidade Técnica Estatal G. I. Nossov, 2011.

SAVEleV, I. V. Curso de Física Geral. In: Óptica, Física Atômica, Física do Núcleo Atômico e Partículas Elementares. [s.1.] Editora Ciência, 1989.

SHIPOLSKY, E. V. Física Atômica. T.1. Sexta ed. Moscou, Rússia: "Escola Superior," 1974.

SIVUKIN, D. A. Curso de Física Geral. T. 5. Física Atômica e Física Nuclear. M. Moscou: Editora Fismatlit, 2008.

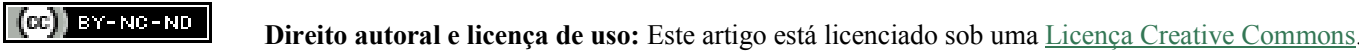

